where $L=$ length of the taper in meters, $\lambda=$ wave length in meters, $R_{t}=$ total resistance of the inner conductor $=R L, Z_{c}=$ characteristic impedance of the uniform line having an outer radius $b$ and an inner radius $a$ of the input of the tapered line, then

$$
T \cong Z_{c} \text {. }
$$

3. If $4 \pi / k \lambda \geqq 6$, we will find that the series for $U$ and $W$ can be approximated by exponentials. In addition, if $R[\exp (k L)-1] / k Z_{c} \geqq 3.5$, then

$$
0.96 \leqq(1-U) /(1-W) \leqq 1.04 .
$$

Since $k L=1.3$, and $\exp (k L)=3.667$, we find that $(1-U) /(1-W)$ is nearly unity if

$$
R_{t} / Z_{c} \geqq 1.7 \text {. }
$$

Thus it can be stated that to satisfy the lower frequency range with a low VSWR, the film length must be long $(L=0.6 \lambda)$, and the total resistance must be near the $Z_{c}$ for extremely low frequencies. In the upper range of frequencies, we should have a total resistance of 1.7 times the characteristic impedance $Z_{c}$. Thus we must conclude for practical reasons, that it would seem more advisable to use the tapered line for frequencies above 1000 megacycles. Of course, if the restriction on the VSWR could be lessened to permit a VSWR of 1.2 or 1.3, then a larger range of frequencies could be covered by the linear tapered line.

\title{
CAPACITY OF A PAIR OF INSULATED WIRES*
}

\section{By W. HOWARD WISE (Bell Telephone Laboratories)}

1. Introduction. This problem has been treated in elegant fashion by Craggs and Tranter. ${ }^{1,2,3}$ Their first two papers employ a conformal transformation of the free space; the third paper works with charge distributions. They end up with an infinite determinant of value zero in which the unknown capacity appears in one element, and conclude that "satisfactory numerical approximations can be obtained by keeping only the first few rows and columns". ${ }^{3}$

This note is written to remark that the end result of a straightforward attack with bi-polar coordinates is

$$
C=\epsilon_{0} / 4\left\{\log \frac{s}{\chi}+\frac{\epsilon_{0}}{\epsilon} \log \frac{\chi}{v}-\sum_{n=1}^{\infty} k_{n}\right\},
$$

where $C=$ capacity, $\epsilon_{0}=$ dielectric constant of air, or other material outside the jackets, $\epsilon=$ dielectric constant of jacket material, $s=$ interaxial separation, $\chi=$ outer

${ }^{*}$ Received Feb. 7, 1949.

1J. W. Craggs and C. J. Tranter, The capacity of twin cable, Q. Appl. Math. 3, 268-272 (1945).

${ }^{2}$ J. W. Craggs and C. J. Tranter, The capacity of twin cable-II, Q. Appl. Math. 3, 380-383 (1946.)

${ }^{3} \mathrm{~J}$. W. Craggs and C. J. Tranter, The capacity of two-dimensional systems of conductors and dielectrics with circular boundaries, Q. J. of Math. (Oxford) 17, 138-144 (1946). 
radius of jackets, $v=$ radius of conductors, and the $k$ are to be obtained from the following set of equations:

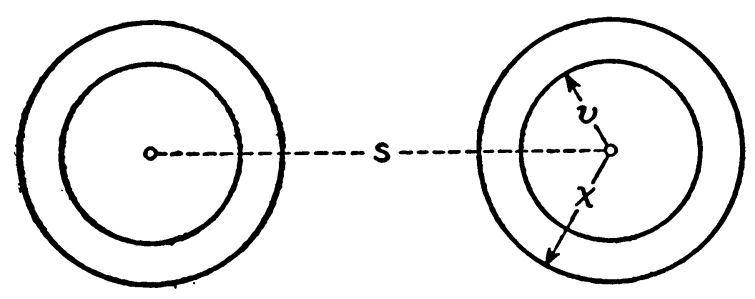

Fig. 1.

$$
\begin{gathered}
m_{1} k_{1}-2 k_{2}-3 k_{3}-4 k_{4}-5 k_{5}-\cdots=1, \\
-k_{1}+m_{2} k_{2}-6 k_{3}-10 k_{4}-15 k_{5}-\cdots=1 / 2, \\
-k_{1}-4 k_{2}+m_{3} k_{3}-20 k_{4}-35 k_{5}-\cdots=1 / 3, \\
-k_{1}-5 k_{2}-15 k_{3}+m_{4} k_{4}-70 k_{5}-\ldots=1 / 4, \\
-k_{1}-6 k_{2}-21 k_{3}-56 k_{4}+m_{5} k_{5}-\ldots=1 / 5, \\
\ldots \ldots \ldots \ldots
\end{gathered}
$$

where

$$
\begin{aligned}
m_{\tau} & =\frac{\epsilon+\epsilon_{0}+\left(\epsilon-\epsilon_{0}\right)(v / \chi)^{2 \tau}}{\epsilon-\epsilon_{0}+\left(\epsilon+\epsilon_{0}\right)(v / \chi)^{2 \tau}}\left(\frac{s}{\chi}\right)^{2 \tau}-\frac{(2 \tau-1) !}{\tau !(\tau-1) !} \\
& =l_{\tau}-(2 \tau-1) ! / \tau !(\tau-1) !
\end{aligned}
$$

The $\tau$-th equation is

$$
l_{\tau} k_{\tau}=\frac{1}{\tau}+\sum_{\kappa=1}^{\infty} \frac{(\tau+\kappa-1) !}{(\kappa-1) ! \tau !} k_{\kappa} .
$$

If one keeps $n$ rows and $n$ columns in the above set of equations, the result is identically the same as that obtained by keeping $n+1$ rows and $n+1$ columns in the infinite determinant in Craggs and Tranter's last paper.

2. Discussion. The series in $k$ converges slowly when the two insulating jackets are in contact. What is worse, if one stops with a small number of $k_{n}$ terms, say five, using (2) as written, the resulting $\sum_{n=1}^{5} k_{n}$ will be too small, not merely because there are only five terms in the series but also because each $k$ is too small. The consequence of all this is that one should start out with the idea of using ten or twelve $k_{n}$ terms. Fortunately, this does not mean that we have to solve ten or twelve simultaneous equations by gradual elimination of the unknowns. Since the $m$ are a rapidly increasing sequence of large numbers, the solution may be arrived at by successive approximations.

If the wires were bare $(\chi=v)$, the series in $k$ would converge more rapidly; but in this case 


$$
\begin{aligned}
& m_{\tau}=(s / v)^{2 \tau}-(2 \tau-1) ! / \tau !(\tau-1) !, \\
& k_{n}=k_{1}^{n} / n \\
& \sum_{n=1}^{\infty} k_{n}=-\log \left(1-k_{1}\right), \\
& k_{1}=\left\{1-\left[1-(2 v / s)^{2}\right]^{1 / 2}\right\} / 2,
\end{aligned}
$$

and (1) reduces to

$$
\begin{aligned}
C & =\epsilon_{0} / 4 \cosh ^{-1}(s / 2 v) \\
& =\epsilon_{0} / 4 \log \left\{s / 2 v+\left[(s / 2 v)^{2}-1\right]^{1 / 2}\right\} .
\end{aligned}
$$

When the two insulating jackets are in contact, $s / \chi=2, s / 2 v=\chi / v$ and

$$
C=\epsilon_{0} / 4\left\{\log 2+\frac{\epsilon_{0}}{\epsilon} \log \frac{\chi}{v}-\sum_{n=1}^{\infty} k_{n}\right\} .
$$

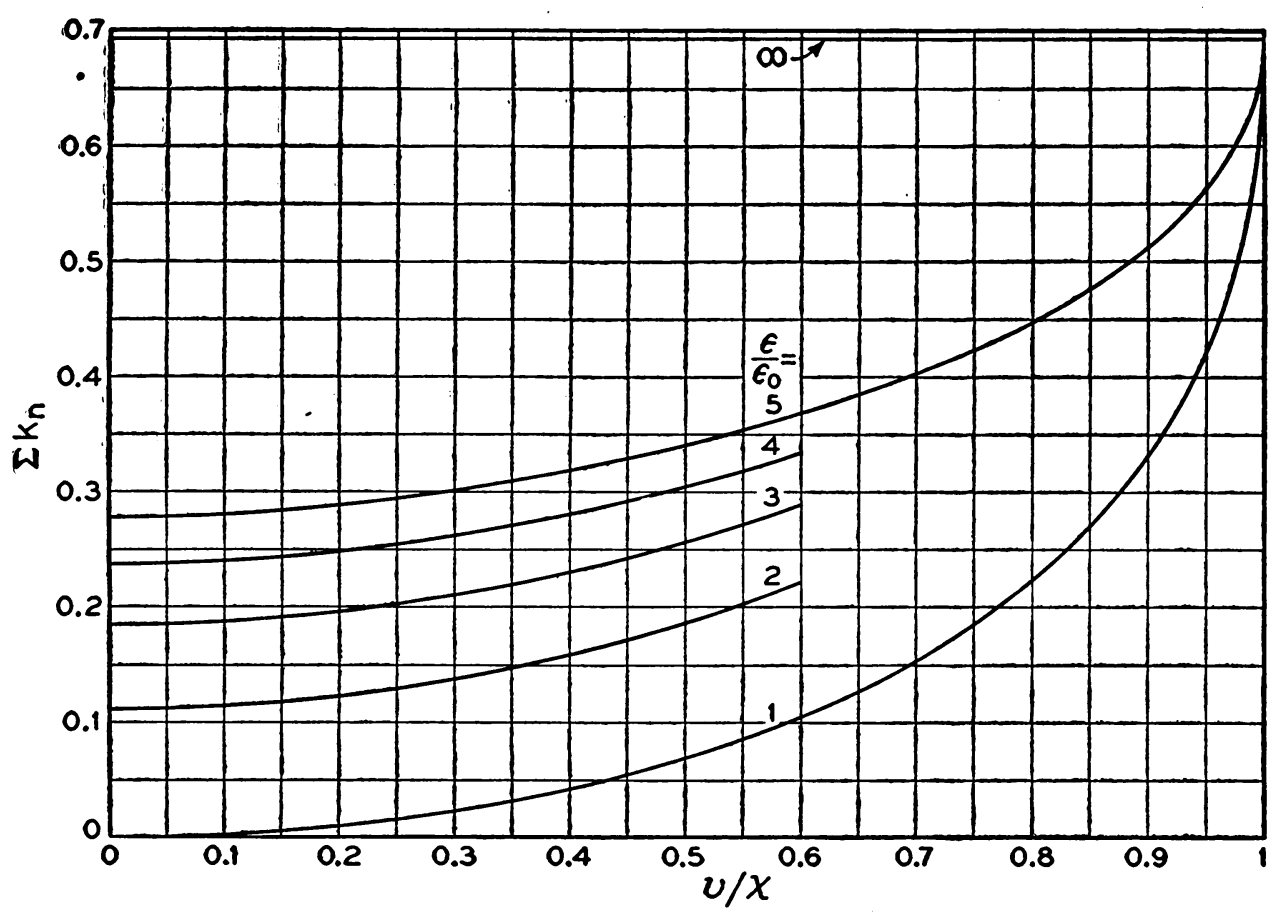

FIg. 2.

The correction term $\sum k_{n}$ in (5) is a function of $\epsilon / \epsilon_{0}$ and $v / \chi$. Figure 2 is a plot of $\sum k_{n}$ versus $v / \chi$ for $\epsilon / \epsilon_{0}=1,2,3,4,5$ and $\infty$. The curve for $\epsilon / \epsilon_{0}=1$ was not obtained by direct computation of the $k$ but by noting that if $\epsilon=\epsilon_{0}$, then

$$
\begin{aligned}
C & =\epsilon_{0} / 4\left[\log s / v-\sum k_{n}\right] \\
& =\epsilon_{0} / 4 \log \left\{s / 2 v+\left[(s / 2 v)^{2}-1\right]^{1 / 2}\right\}
\end{aligned}
$$


and so

$$
\sum k_{n}=\log 2-\log \left\{1+\left[1-(v / x)^{2}\right]^{1 / 2}\right\} .
$$

The upper end of the curve for $\epsilon / \epsilon_{0}=5$ was fixed by noting that if $v / \chi$ is close to unity then $k_{n} \approx k_{1}^{n} / n,\left(m_{1}+1\right) k_{1} \approx 1 /\left(1-k_{1}\right), k_{1} \approx\left\{1-\left[1-4 /\left(m_{1}+1\right)\right]^{1 / 2}\right\} / 2$ and $\sum k_{n} \approx \log 1 /\left(1-k_{1}\right) \approx \log 2 /\left\{1+\left[1-4 /\left(m_{1}+1\right)\right]^{1 / 2}\right\}$.

With $v / \chi=0$ it is easy to solve (3) by the method of successive substitutions, thus getting

where

$$
k_{n}=g \frac{\rho^{2 n}}{n}\left\{1+g\left[\left(p_{0} / p_{1}\right)^{n}-1\right]+\sum_{\imath=2}^{\infty} g^{\iota}\left[\left(p_{\iota-1} / p_{\iota}\right)^{n}-\left(p_{\iota-2} / p_{\imath-1}\right)^{n}\right]\right\},
$$

$$
\begin{aligned}
& g=\left(\epsilon-\epsilon_{0}\right) /\left(\epsilon+\epsilon_{0}\right), \\
& \rho=\chi / s, \\
& p_{0}=1, \quad p_{1}=1-\rho^{2}, \quad p_{2}=1-2 \rho^{2}, \cdots, \quad p_{\imath}=p_{\imath-1}-\rho^{2} p_{\imath-2} ; \\
& \sum_{n=1}^{\infty} k_{n}=-g \log p_{1}-\sum_{\imath=2}^{\infty} g^{\imath} \log \left(p_{\imath} p_{\imath-2} / p_{\imath-1}^{2}\right) .
\end{aligned}
$$

With $\rho=1 / 2$ we have

$$
\sum_{n=1}^{\infty} k_{n}=-\sum_{n=2}^{\infty} g^{n-1} \log \frac{n^{2}-1}{n^{2}} .
$$

This formula was used to check the more laborious computations based on (2) at $v / \chi=0$.

Since $l_{\tau}=(s / v)^{2 \tau}$ if $\epsilon=\epsilon_{0},(7)$ with $g=1$ and $\rho=v / s$ gives $\sum k_{n}$ for bare wires. Thus

$$
\begin{aligned}
C & =\epsilon_{0} / 4\left\{\log \frac{1}{\rho}+\log p_{1}+\sum_{\imath=2}^{\infty} \log p_{\iota} p_{\imath-2} / p_{\imath-1}^{2}\right\} \\
& =\epsilon_{0} / 4\left\{\log \frac{1}{\rho}+\log \lim _{\imath \rightarrow \infty} p_{\imath} / p_{\imath-1}\right\} \\
& =\epsilon_{0} / 4\left\{\log \frac{1}{\rho}+\log \frac{1}{2}\left\{1+\left[1-4 \rho^{2}\right]^{1 / 2}\right\}\right. \\
& =\epsilon_{0} / 4 \log \left\{s / 2 v+\left[(s / 2 v)^{2}-1\right]^{1 / 2}\right\} .
\end{aligned}
$$

It is easy enough to write out a solution of (3) by the method of successive substitutions and so find that

$$
\begin{aligned}
\sum_{n=1}^{\infty} k_{n}=\sum_{n=1}^{\infty} & \frac{1}{l_{n} n}+\sum_{n=1}^{\infty} \frac{1}{l_{n}} \sum_{\kappa=1}^{\infty} C_{\kappa-1}^{n+\kappa-1} \frac{1}{l_{\kappa} \kappa} \\
& +\sum_{n=1}^{\infty} \frac{1}{l_{n}} \sum_{\kappa=1}^{\infty} C_{\kappa-1}^{n+\kappa-1} \frac{1}{l_{k}} \sum_{\lambda=1}^{\infty} C_{\lambda-1}^{\kappa+\lambda-1} \frac{1}{l_{\lambda} \lambda} \\
& +\sum_{n=1}^{\infty} \frac{1}{l_{n}} \sum_{\kappa=1}^{\infty} C_{\kappa-1}^{n+\kappa-1} \frac{1}{l_{k}} \sum_{\lambda=1}^{\infty} C_{\lambda-1}^{\kappa+\lambda-1} \frac{1}{l_{\lambda}} \sum_{\mu=1}^{\infty} C_{\mu-1}^{\lambda+\mu-1} \frac{1}{l_{\mu} \mu}+\cdots ;
\end{aligned}
$$


but, aside from the simple case $v / \chi=0$ discussed above, (9) seems to be of no help. It is equally easy to write out the Fredholm type of solution of (3),

$$
l_{\iota} k_{\iota}=\frac{1}{\iota}+\frac{1}{\Delta} \sum_{\kappa=1}^{\infty} \frac{1}{\kappa} \Delta_{\iota \kappa},
$$

where

$$
\begin{gathered}
\Delta=1-\sum_{\iota=1}^{\infty} K_{\iota \iota}+\frac{1}{2 !} \sum_{\iota=1}^{\infty} \sum_{\kappa=1}^{\infty}\left|\begin{array}{ll}
K_{\iota \iota} & K_{\iota \kappa} \\
K_{\kappa \iota} & K_{\kappa \kappa}
\end{array}\right|-\cdots, \\
\Delta_{\iota \kappa}=K_{\iota \kappa}-\sum_{n=1}^{\infty}\left|\begin{array}{cc}
K_{\iota \kappa} & K_{\iota n} \\
K_{n \kappa} & K_{n n}
\end{array}\right|+\frac{1}{2 !} \sum_{n=1}^{\infty} \sum_{m=1}^{\infty}\left|\begin{array}{ccc}
K_{\iota \kappa} & K_{\iota n} & K_{\iota m} \\
K_{n \kappa} & K_{n n} & K_{n m} \\
K_{m \kappa} & K_{m n} & K_{m m}
\end{array}\right|-\cdots,
\end{gathered}
$$

and

$$
K_{\iota \kappa}=C_{\kappa-1}^{\iota+\kappa-1} / l_{\kappa}
$$

but no use has been found for it.

3. Numerical example illustrating convergence.

$$
s=2 \chi, \quad v / \chi=.4, \quad \epsilon / \epsilon_{0}=4 .
$$

If we assume that $1,2,3,4$ and $10 k$ terms are enough, we compute in turn, $k_{1}=$ $.2097, \sum_{n=1}^{2} k_{n}=.2544, \sum_{n=1}^{3} k_{n}=.2692, \sum_{n=1}^{4} k_{n}=.2751$ and $\sum_{n=1}^{10} k_{n}=.2807$. The sum of the first four of the $k_{n}$ in $\sum_{n=1}^{10} k_{n}$ is .2797 . The late terms in the sum are more important indirectly in computing the earlier terms than in the sum itself.

\section{THE TEMPERATURE IN AN ACCRETING MEDIUM WITH HEAT GENERATION*}

By A. E. BENFIELD (Cruft Laboratory, Harvard University)

The thermal problem of this note was solved with the hope of using it to try to test the theory that the earth was formed by accretion on the dust cloud hypothesis; ${ }^{1}$ but there are many uncertain and unknown physical factors involved and, on reflection, it seems that the contemplated thermal considerations are unable at present to help in drawing conclusions. However, it is hoped that the following mathematical solution may be of interest and aid to others having related problems involving less uncertain physical conditions.

As the spherical case presents some difficulties, we shall merely consider here a

*Received March 25, 1949.

${ }^{1}$ See, for instance, F. L. Whipple, Scientific American 178, 34 (1948). 